\title{
Interacciones Moleculares de la Mezcla De [Bmim][TfO] + Agua a Partir de Datos de Viscosidad
}

\author{
Manuel S. Páez ${ }^{(1) \star}$, María K. Vergara ${ }^{(1)}$, Omar A. Pérez $^{(2)}$ \\ (1) Universidad de Córdoba. Departamento de Química. Facultad de Ciencias Básicas. \\ Carrera 6 No. 76-103. Km 3. Vía Cereté. Córdoba. Colombia. (e-mail: mspaezm@unal.edu.co) \\ (2) Universidad de Córdoba. Departamento de Ingeniería de Alimentos. Facultad de Ciencias Agrícolas. \\ Carrera 6 No. 76-103. Km 3. Vía Cereté. Córdoba. Colombia.
}

${ }^{*}$ Autor a quien debe dirigirse la correspondencia.

Recibido Mar. 31, 2014; Aceptado Jun. 18, 2014; Versión final recibida Jul. 9, 2014

\section{Resumen}

Se determinaron los tiempos de flujo de la mezcla binaria del líquido iónico trifluorometanosulfonato de 1Butil-3-metil imidazolio + agua en el intervalo de temperaturas de 283.15 a $318.15 \mathrm{~K}$ usando un microviscosímetro Anton Paar (AMVn). A partir de los datos obtenidos se calcularon las viscosidades absolutas, los coeficientes A, B y D del modelo de Jones y Dole para la viscosidad, y los parámetros de activación del flujo viscoso. Esto con el objetivo de obtener información acerca de las interacciones presentes en solución. Los valores $\partial B / \partial T$ de la mezcla binaria son negativos, indicando que las interacciones ionsolvente no son favorecidas y que el efecto formador ejercido por la parte no polar del liquido iónico sobre la estructura del agua es favorecido. El proceso de flujo viscoso resultó endotérmico y se mostró en concordancia con el signo dado por la ley de Raoult.

Palabras clave: viscosidad, interacciones moleculares, parámetros de activación, líquido iónico, [Bmim] [TfO]

\section{Molecular Interactions of the Mixture of [Bmim] [TfO] + Water from Viscosity Data}

\begin{abstract}
Flow times of the binary mixture ionic liquid 1-Butyl-3-methyl imidazolium trifluoromethanesulfonate + water were determined in the temperature range 283.15 to $318.15 \mathrm{~K}$ using an Anton Paar (AMVn) microviscometer. The absolute viscosity, the coefficients $A, B$ and $D$ Jones and Dole model for the viscosity, and the activation parameters of viscous flow were calculated from the data. This was done to obtain information about the interactions present in solution. The $\partial B / \partial T$ values of the binary mixture are negative, indicating that the ion-solvent interactions are not favored and the forming effect exerted by the non-polar part of the ionic liquid on the water structure is favored. The viscous flow process was endothermic and showed agreement with the sign given by Raoult's law.
\end{abstract}

Keywords: viscosity, molecular interactions, activation parameters, ionic liquid, [Bmim] [TfO] 


\section{INTRODUCCIÓN}

Los líquidos iónicos constituyen una de las nuevas familias de materiales más prometedores para el desarrollo de la nueva industria de la Química verde, ya que con su uso se disminuye la contaminación ambiental (Cabeza et al, 2011). A diferencia de los disolventes orgánicos tradicionales, los líquidos iónicos se encuentran constituidos exclusivamente por iones, a saber un catión de naturaleza orgánica y un anión orgánico o inorgánico. Estos pueden ser diseñados de tal forma que las sales resultantes no se pueden empaquetar de forma compacta y como consecuencia, dichas sustancias no pueden cristalizar fácilmente y permanecen en estado líquido dentro de un amplio rango de temperaturas, incluso a temperatura ambiente como es el caso del [Bmim][OTF] (Bernal, 2013).

Los estudios sobre la viscosidad de soluciones electrolíticas, tales como la mezcla binaria del líquido iónico (LI) [Bmim][OTF] son útiles, ya que estos líquidos tienen gran aplicación en diversas ramas de la industria química, debido a que pueden actuar como catalizadores y/o disolventes, lo que hace que tengan gran interés como fluidos para aplicaciones de ingeniería, por lo que son llamados solventes de diseño debido a que están formados por cationes y aniones que conducen a la realización de tareas específicas (Páez et al., 2013; Cortés et al., 2010; Shamsipur et al.,2010).Estas aplicaciones requieren de datos de propiedades termodinámicas y termofísicas tales como la densidad, viscosidad, equilibrio de fases de mezclas binarias entre un líquido iónico y un solvente molecular entre otras (Pasos et al., 2014; Khan et al., 2014; Faúndez et al., 2013; Martínez et al., 2011)

En este trabajo se reportan datos de viscosidades dinámicas $(\eta)$ de la mezcla binaria del líquido iónico [Bmim][OTF] + agua a una presión atmosférica de 1.011 bar, a las temperaturas de 283.15-318.15 K a diferentes concentraciones. Los valores experimentales de $\eta$ fueron usados para calcular las desviaciones de viscosidad, los coeficientes de Redlich-Kister, los coeficientes A, B y D de viscosidad y los parámetros de activación del flujo viscoso. El comportamiento de estos parámetros con la concentración y la temperatura fue analizado en términos de las interacciones que ocurren a nivel de la solución.

\section{MATERIALES Y MÉTODOS}

El líquido iónico trifluorometanosulfonato de 1-Butil-3-metil imidazolio fue obtenido de Across Organic con una pureza $\geq 99 \%$. El contenido de agua de este LI fue de $15 \mathrm{ppm}$, y se determinó usando el método de Karl-Fisher. Para reducir el contenido de agua y los compuestos volátiles que tienen influencia sobre las propiedades fisicoquímicas de las soluciones; éste, se secó con agitación a temperatura moderada $(T=343$ $\mathrm{K})$ y a baja presión $(\mathrm{p}=0,2 \mathrm{~Pa})$ durante al menos $48 \mathrm{~h}$ antes de su uso. Después del secado del líquido iónico es guardado en botellas bajo un gas inerte. El agua usada para preparar las soluciones fue bidestilada y desgasificada y presentó una conductividad menor que $2 \mu \mathrm{S}$. La preparación de las soluciones del sistema acuoso [Bmim][OTF]+agua, se realizó mediante el método gravimétrico usando una balanza (OHAUS, modelo Explorer con una incertidumbre de $\pm 1 \times 10^{-4} \mathrm{~g}$ en botellas herméticamente cerradas para evitar pérdida por evaporación de alguno de los componentes. Los tiempos de flujo del líquido puro y las soluciones se midieron usando un microviscosímetro Anton Paar modelo AMVn. La viscosidad se obtuvo con una incertidumbre de $\pm 0.001 \mathrm{mPa}$ s en el intervalo de temperaturas (283.15-318.15) $\mathrm{K}$.

\section{RESULTADOS Y DISCUSIÓN}

Las viscosidades del líquido iónico puro experimentales se muestran en el gráfico de desviaciones, Figura 1 comparado con los valores de literatura;

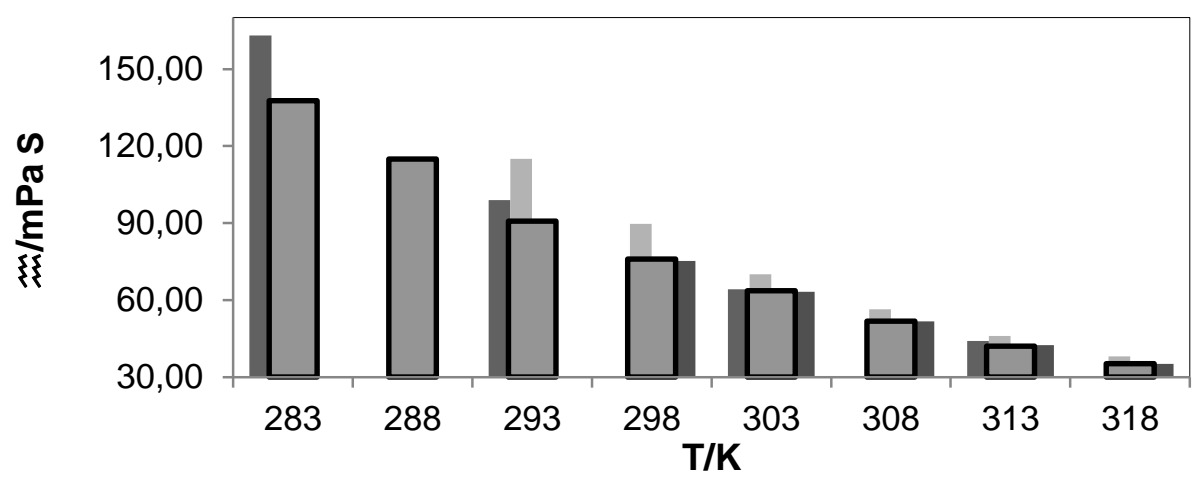

Fig. 1: Comparación de datos de viscosidad $\eta$ de [Bmim][OTF] experimentales ( $\square$ ) y los reportados por las referencias,( $\square$ Seddon et al., 2002; Seoane et al.,2012; Shamsipur et al., 2010; Ge et al., 2008) 
En la figura 1, se observa que hay mayor coincidencia entre los datos reportados en literatura y los obtenidos experimentalmente, sobre todo en las temperaturas altas donde existe el mayor número de reportes bibliográficos, tal y como se observa para 303.15 y $313.15 \mathrm{~K}$ donde los valores son muy próximos entre sí. A la temperatura de $288,15 \mathrm{~K}$ no se observan comparaciones, debido a que en las referencias consultadas no hay reportes a esta temperatura. En la Tabla 1, se presentan los resultados experimentales del sistema [Bmim][OTF] + agua obtenidos para las viscosidades dinámicas en función de la temperatura y la concentración. Se observa que la adición del líquido iónico [Bmim][OTF] al agua logra incrementar la viscosidad de esta. También se aprecia que la viscosidad de las soluciones de [Bmim][OTF] + agua disminuye con el incremento de la temperatura, lo cual es una consecuencia de la perturbación de la red espacial en la solución por el movimiento térmico de las moléculas (Páez et al.,2014). A partir de los datos de la Tabla 1, se determinó la viscosidad relativa como la razón de la viscosidad dinámica de la solución ( $\eta$ ) respecto a la del solvente puro $\left(\eta_{0}\right)$ en el intervalo de concentración molar (0.0100-3.9662) $\mathrm{mol} \mathrm{L}^{-1}$, estas viscosidades se ajustaron a partir de la ecuación Jones-Dole para soluciones electrolíticas concentradas,(Kaminsky, 1957):

$$
\eta_{r}=\frac{\eta}{\eta_{0}}=1+A \sqrt{C}+B C+D C^{2}
$$

Tabla 1: Viscosidades dinámicas de la mezcla [Bmim][OTF] + agua a temperaturas de 283.15 a $318.15 \mathrm{~K}$

\begin{tabular}{|c|c|c|c|c|c|c|c|c|}
\hline $\mathrm{T} / \mathrm{K}$ & 283.15 & 288.15 & 293.15 & 298.15 & 303.15 & 308.15 & 313.15 & 318.15 \\
\hline$x_{2}$ & \multicolumn{8}{|c|}{$\eta /(\mathrm{mPa} s)$} \\
\hline 0.0000 & 1.304 & 1.137 & 1.002 & 0.890 & 0.797 & 0.719 & 0.653 & 0.597 \\
\hline 0.0002 & 1.321 & 1.150 & 1.014 & 0.904 & 0.806 & 0.725 & 0.656 & 0.601 \\
\hline 0.0004 & 1.329 & 1.157 & 1.020 & 0.909 & 0.810 & 0.729 & 0.659 & 0.604 \\
\hline 0.0005 & 1.338 & 1.164 & 1.025 & 0.914 & 0.814 & 0.733 & 0.662 & 0.607 \\
\hline 0.0009 & 1.354 & 1.178 & 1.037 & 0.924 & 0.823 & 0.740 & 0.667 & 0.613 \\
\hline 0.0014 & 1.379 & 1.199 & 1.055 & 0.939 & 0.836 & 0.751 & 0.677 & 0.621 \\
\hline 0.0018 & 1.397 & 1.214 & 1.067 & 0.950 & 0.844 & 0.759 & 0.683 & 0.627 \\
\hline 0.0036 & 1.480 & 1.283 & 1.125 & 1.000 & 0.887 & 0.795 & 0.713 & 0.655 \\
\hline 0.0054 & 1.565 & 1.353 & 1.184 & 1.051 & 0.929 & 0.832 & 0.745 & 0.683 \\
\hline 0.0072 & 1.648 & 1.422 & 1.242 & 1.102 & 0.971 & 0.869 & 0.777 & 0.712 \\
\hline 0.0089 & 1.731 & 1.491 & 1.300 & 1.151 & 1.013 & 0.905 & 0.809 & 0.739 \\
\hline 0.0107 & 1.815 & 1.561 & 1.359 & 1.202 & 1.056 & 0.942 & 0.842 & 0.768 \\
\hline 0.0142 & 1.984 & 1.700 & 1.475 & 1.303 & 1.140 & 1.015 & 0.910 & 0.824 \\
\hline 0.0160 & 2.067 & 1.769 & 1.533 & 1.353 & 1.182 & 1.052 & 0.945 & 0.852 \\
\hline 0.0177 & 2.151 & 1.838 & 1.592 & 1.404 & 1.224 & 1.089 & 0.981 & 0.880 \\
\hline 0.0826 & 5.563 & 4.998 & 4.246 & 3.644 & 3.107 & 2.813 & 2.545 & 2.318 \\
\hline 0.2127 & 13.052 & 11.583 & 9.700 & 8.203 & 6.884 & 6.102 & 5.910 & 5.309 \\
\hline 0.3105 & 23.755 & 21.804 & 17.650 & 13.749 & 11.385 & 10.450 & 8.797 & 7.915 \\
\hline 0.3864 & 31.730 & 28.912 & 23.443 & 18.327 & 15.145 & 13.774 & 11.627 & 10.220 \\
\hline 0.4476 & 39.024 & 35.324 & 28.689 & 22.506 & 17.309 & 16.732 & 14.161 & 12.322 \\
\hline 0.5741 & 57.136 & 50.754 & 41.406 & 32.794 & 26.902 & 23.017 & 19.525 & 16.844 \\
\hline 0.6179 & 64.447 & 56.779 & 46.415 & 36.921 & 30.213 & 25.621 & 21.730 & 18.718 \\
\hline 0.6845 & 76.652 & 66.471 & 52.009 & 42.508 & 35.420 & 29.624 & 25.132 & 21.236 \\
\hline 0.7297 & 85.511 & 73.172 & 57.811 & 46.011 & 38.724 & 32.432 & 27.139 & 22.944 \\
\hline 0.7825 & 95.957 & 80.600 & 63.991 & 52.194 & 43.807 & 35.959 & 29.835 & 25.040 \\
\hline 0.8169 & 102.308 & 84.798 & 67.396 & 56.001 & 46.359 & 38.016 & 31.528 & 26.739 \\
\hline 1.0000 & 137.500 & 115.000 & 90.800 & 76.000 & 63.600 & 51.729 & 42.120 & 35.174 \\
\hline
\end{tabular}

donde $C$ es la molaridad $\left(\mathrm{mol} \mathrm{L}^{-1}\right), \eta_{r}$ es la viscosidad relativa, $\mathrm{A}\left(\mathrm{L}^{1 / 2} \mathrm{~mol}^{-1 / 2}\right)$ coeficiente de Falkenhagen que mide las interacciones de largo alcance ión-ión mediadas por el solvente, $\mathrm{B}\left(\mathrm{L} \mathrm{mol}{ }^{-1}\right)$ es una constate que tiene en cuenta las interacciones de corto alcance ion-solvente y solvente-solvente, por consiguiente proporciona información acerca de la hidratación de solutos y los efectos en la estructura de las moléculas del solvente situadas en las esferas de hidratación del soluto y el coeficiente $D$ incluye todas las interacciones estructurales ión-solvente e ión-ión que no se toman en cuente en los términos $A \sqrt{C}$ y $B C$. La teoría de Falkenhagen permite el cálculo de la constante $A$ en el caso de iguales valencias iónicas $\left(z_{1}=Z_{2}=z\right)$ e iguales movilidades iónicas límites $\left(L_{1}^{\infty}=L_{2}^{\infty}=L^{\infty}\right)$, mediante la ecuación. 


$$
A=\frac{0.517 z^{2}}{L^{\infty} \sqrt{\varepsilon_{0}} \eta_{0} T}
$$

donde $\varepsilon_{0}$ es la constante dieléctrica del solvente y T es la temperatura absoluta.

En la Tabla 2, se observa que el coeficiente B es mucho mayor que $A$ y $D$, lo cual indica que las interacciones dominantes en este sistema se pueden atribuir a débiles interacciones ión-agua y fuertes interacciones agua-agua de corto alcance, mientras el signo negativo en $\partial B / \partial T$, podría indicar que los iones del líquido iónico [Bmim][OTF] están débilmente hidratados, corroborando con ello el comportamiento hidrofóbico de las partes apolares de los iones del líquido iónico (Portacio et al., 2011). Este resultado es consistente con el análisis de las propiedades volumétricas de este mismo sistema (Páez et al.,2013) y con los resultados positivos de los coeficientes de actividad obtenidos por ELV a 298.15K (Pasos et al., 2014; Khan et al., 2014). Por otra parte, el valor del coeficiente D podría indicar el favorecimiento de autointeracciones iónicas e interacciones entre iones de signos opuestos no contempladas en los términos $A \sqrt{C}$ y $B C$ (Zhao, 2006).

Por otra parte, se procedió a calcular la viscosidad absoluta $(\eta)$ de este sistema al considerarlo un fluido Newtoniano, siguiendo la aproximación de Eyring con ayuda de la ecuación (3) (Páez et al., 2012)

$$
\eta=\frac{h N}{V_{m}} \exp \left(\frac{\Delta G^{*}}{R T}\right)=\frac{h N}{V_{m}} \exp \left(\frac{\Delta H^{*}}{R T}-\frac{\Delta S^{*}}{R}\right)
$$

donde $h$ es la constante de Planck, $N$ es número de Avogadro, $V_{m}$ es el volumen molar del líquido, T es la temperatura absoluta, y $\Delta G^{\neq}, \Delta H^{\neq}, \Delta S^{\ddagger}$ son la energía libre de Gibbs, la entalpía y la entropía de activación del flujo viscoso respectivamente. $\Delta H^{\neq}$y $\Delta S^{\neq}$se obtienen de la pendiente $\Delta H^{\neq} / R$ y el intercepto $-\Delta S^{\neq} / R$ de la gráfica de $\ln \eta V_{m} / h N$ vs $1 / T$; mientras $\Delta G^{\neq}$, se obtiene mediante la ecuación (4):

$\Delta G^{\neq}=\Delta H^{\neq}-T \Delta S^{*}$

Tabla 2: Coeficientes $A, B$ y $D$ de viscosidad y $\partial B / \partial T$ de la mezcla binaria de $[$ Bmim] $[\mathrm{OTF}]+$ agua a diferentes temperaturas.

\begin{tabular}{cccccc}
\hline $\begin{array}{c}\mathrm{T} \\
(\mathrm{K})\end{array}$ & $\begin{array}{c}\mathrm{A} \\
\left(\mathrm{L}^{1 / 2} \mathrm{~mol}^{-1 / 2}\right)\end{array}$ & $\begin{array}{c}\mathrm{B} \\
\left(\mathrm{L} \mathrm{mol}^{-1}\right)\end{array}$ & $\begin{array}{c}\mathrm{D} \\
\left(\mathrm{L}^{2} \mathrm{~mol}^{-2}\right)\end{array}$ & $\begin{array}{c}\mathrm{dB} / \mathrm{dT} \\
(\mathrm{L} \mathrm{mol}\end{array}$ & \\
\hline 283.15 & $4.29 \mathrm{E}-03$ & 0.632 & 0.043 & -0.081 & $8.6 \mathrm{E}-03$ \\
288.15 & $4.80 \mathrm{E}-03$ & 0.582 & 0.064 & -0.082 & $1.1 \mathrm{E}-02$ \\
293.15 & $5.34 \mathrm{E}-03$ & 0.557 & 0.061 & -0.083 & $1.1 \mathrm{E}-02$ \\
298.15 & $5.90 \mathrm{E}-03$ & 0.536 & 0.054 & -0.085 & $2.9 \mathrm{E}-07$ \\
303.15 & $6.45 \mathrm{E}-03$ & 0.511 & 0.052 & -0.088 & $9.3 \mathrm{E}-03$ \\
308.15 & $7.01 \mathrm{E}-03$ & 0.482 & 0.061 & -0.092 & $8.9 \mathrm{E}-03$ \\
313.15 & $7.58 \mathrm{E}-03$ & 0.453 & 0.068 & -0.097 & $1.7 \mathrm{E}-07$ \\
318.15 & $8.11 \mathrm{E}-03$ & 0.413 & 0.073 & -0.103 & $1.1 \mathrm{E}-07$ \\
\hline
\end{tabular}

Los resultados que se obtienen después de realizar este tratamiento se muestran en la Tabla 3 , observándose que los valores de $\Delta H^{\neq}, \Delta S^{\neq}$y $\Delta G^{\neq}$son positivos en todo el rango de concentración, de igual manera se aprecia una disminución de la energía libre de activación con el aumento de la temperatura. De acuerdo con la teoría de Eyring el cambio de la energía de Gibbs de activación $\Delta G^{\neq}$, controla la velocidad de flujo en el líquido, la cual se rige por la capacidad de los iones que constituyen al LI para moverse en las cavidades y por la facilidad con que las cavidades están dispuestas en el agua. Ahora, como $\Delta G^{\neq}$se incrementa con la concentración del LI, ello evidencia una mayor dificultad en el movimiento de los iones a través del disolvente, resultado de la complejidad de las interacciones características del sistema en estudio: interacciones débiles de corto alcance ion-solvente y fuertes interacciones agua-agua presentes en las esferas de hidratación (agua estructurada), interacciones entre iones de signo opuesto y autointeracciones entre los iones $\left[\mathrm{Bmim}^{+}\right]-\left[\mathrm{Bmim}^{+}\right]$y $\left[\mathrm{CF}_{3} \mathrm{SO}_{3}^{-}\right]-\left[\mathrm{CF}_{3} \mathrm{SO}_{3}^{-}\right]$. Los valores positivos para la variación de entropía de activación $\Delta S^{\neq}$muestran que las especies que forman el estado activado son más desordenadas que el estado inicial. Mientras que los valores de $\Delta H^{\neq}$para las mezclas son positivos y se incrementan con la concentración del LI, ello podría indicar que el proceso de flujo viscoso es endotérmico y como también un incremento de las interacciones entre especies similares. Este último resultado resulta interesante ya que en general para las soluciones como regla el signo de la desviación de la ley de Raoult y el signo del calor de mezcla deben coincidir. 
Tabla 3: Parámetros de activación $\Delta H^{\ddagger}, \Delta S^{\ddagger}$ y $\Delta G^{\neq}$para la mezcla [Bmim][OTF]+agua a varias temperaturas

\begin{tabular}{|c|c|c|c|c|c|c|c|c|c|c|}
\hline \multirow{2}{*}{\multicolumn{3}{|c|}{ [Bmim][OTF]+ Agua }} & \multicolumn{8}{|c|}{$\mathrm{T} / \mathrm{K}$} \\
\hline & & & \multirow{2}{*}{283.15} & \multirow[t]{2}{*}{288.15} & \multirow{2}{*}{293.15} & \multirow{2}{*}{$\begin{array}{r}298.15 \\
\Delta G^{\neq} / \mathrm{k}\end{array}$} & \multirow{2}{*}{$\begin{array}{l}303.15 \\
\mathrm{~J} \mathrm{~mol}^{-1}\end{array}$} & \multirow[t]{2}{*}{308.15} & \multirow[t]{2}{*}{313.15} & \multirow[t]{2}{*}{318.15} \\
\hline $\mathrm{m} / \mathrm{kgmol}^{-1}$ & $\Delta H^{\neq} / \mathrm{kJmol}^{-1}$ & $\Delta S^{\neq} \mathrm{Jmol}^{-1} \mathrm{~K}^{-1}$ & & & & & & & & \\
\hline 0.0101 & 16.65 & 24.88 & 9.61 & 9.48 & 9.36 & 9.23 & 9.11 & 8.99 & 8.86 & 8.74 \\
\hline 0.0201 & 16.69 & 24.92 & 9.63 & 9.51 & 9.38 & 9.26 & 9.14 & 9.01 & 8.89 & 8.76 \\
\hline 0.0302 & 16.73 & 24.99 & 9.65 & 9.53 & 9.40 & 9.28 & 9.15 & 9.03 & 8.90 & 8.78 \\
\hline 0.0500 & 16.80 & 25.11 & 9.69 & 9.57 & 9.44 & 9.31 & 9.19 & 9.06 & 8.94 & 8.81 \\
\hline 0.0800 & 16.91 & 25.28 & 9.75 & 9.62 & 9.50 & 9.37 & 9.24 & 9.12 & 8.99 & 8.86 \\
\hline 0.1005 & 16.98 & 25.40 & 9.79 & 9.66 & 9.53 & 9.41 & 9.28 & 9.15 & 9.03 & 8.90 \\
\hline 0.2001 & 17.29 & 25.85 & 9.97 & 9.84 & 9.71 & 9.58 & 9.45 & 9.32 & 9.19 & 9.06 \\
\hline 0.3009 & 17.56 & 26.21 & 10.14 & 10.01 & 9.88 & 9.75 & 9.62 & 9.49 & 9.36 & 9.22 \\
\hline 0.4006 & 17.80 & 26.46 & 10.31 & 10.17 & 10.04 & 9.91 & 9.78 & 9.64 & 9.51 & 9.38 \\
\hline 0.4993 & 18.01 & 26.66 & 10.46 & 10.33 & 10.19 & 10.06 & 9.93 & 9.79 & 9.66 & 9.53 \\
\hline 0.6001 & 18.20 & 26.78 & 10.61 & 10.48 & 10.35 & 10.21 & 10.08 & 9.94 & 9.81 & 9.68 \\
\hline 0.8001 & 18.52 & 26.90 & 10.90 & 10.76 & 10.63 & 10.50 & 10.36 & 10.23 & 10.09 & 9.96 \\
\hline 0.9000 & 18.64 & 26.88 & 11.03 & 10.90 & 10.76 & 10.63 & 10.50 & 10.36 & 10.23 & 10.09 \\
\hline 1.0006 & 18.76 & 26.82 & 11.16 & 11.03 & 10.90 & 10.76 & 10.63 & 10.49 & 10.36 & 10.22 \\
\hline 5.0004 & 18.74 & 14.79 & 14.55 & 14.48 & 14.41 & 14.33 & 14.26 & 14.18 & 14.11 & 14.04 \\
\hline 14.9983 & 19.27 & 5.01 & 17.85 & 17.83 & 17.80 & 17.78 & 17.75 & 17.73 & 17.70 & 17.68 \\
\hline 25.0018 & 24.18 & 14.69 & 20.02 & 19.95 & 19.88 & 19.80 & 19.73 & 19.66 & 19.58 & 19.51 \\
\hline 34.9582 & 24.69 & 12.60 & 21.12 & 21.06 & 20.99 & 20.93 & 20.87 & 20.80 & 20.74 & 20.68 \\
\hline 44.9784 & 25.16 & 11.60 & 21.88 & 21.82 & 21.76 & 21.70 & 21.65 & 21.59 & 21.53 & 21.47 \\
\hline 74.8296 & 26.54 & 11.43 & 23.30 & 23.24 & 23.19 & 23.13 & 23.07 & 23.01 & 22.96 & 22.90 \\
\hline 89.7558 & 26.82 & 10.91 & 23.73 & 23.68 & 23.62 & 23.57 & 23.51 & 23.46 & 23.40 & 23.35 \\
\hline 120.4253 & 27.35 & 10.75 & 24.31 & 24.25 & 24.20 & 24.15 & 24.09 & 24.04 & 23.98 & 23.93 \\
\hline 149.8510 & 28.01 & 11.69 & 24.70 & 24.64 & 24.58 & 24.52 & 24.46 & 24.40 & 24.35 & 24.29 \\
\hline 199.6805 & 28.38 & 11.53 & 25.11 & 25.05 & 25.00 & 24.94 & 24.88 & 24.82 & 24.77 & 24.71 \\
\hline 247.6955 & 28.35 & 10.62 & 25.34 & 25.29 & 25.23 & 25.18 & 25.13 & 25.07 & 25.02 & 24.97 \\
\hline
\end{tabular}

\section{CONCLUSIONES}

En este artículo se reportan las viscosidades de las soluciones acuosas de [Bmim][OTF] en función de la concentración y la temperatura. Se encontró que las fuerzas débiles de corto alcance ión-solvente y las interacciones fuertes entre especies disimilares, dominan el comportamiento viscosimétrico del sistema [Bmim+][OTF] + agua, en todo el intervalo de concentración molar a todas las temperaturas de trabajo. El proceso de flujo viscoso resultó endotérmico y se mostró en concordancia con el signo de la ley de Raoult. El signo de $\partial B / \partial T$ resultó negativo indicando con ello el carácter hidrofóbico de las partes apolares de los iones de este líquido iónico.

\section{AGRADECIMIENTOS}

Los autores agradecen a la universidad de Córdoba por el apoyo prestado para la realización de este trabajo.

\section{REFERENCIAS}

Bernal, B., Tecnología enzimática para la sacarificación de biomasas vegetales en solventes Neotéricos, tesis de doctorado, Universidad de Murcia, Facultad de química, Murcia, España (2013).

Cabeza, $\mathrm{O}$ y otros cinco autores, Physical properties of binary mixtures of iLs with water and ethanol, $A$ review, Cap. 5 en Ionic Liquids: Theory, Properties, New Approaches, Alexander Kokorin (Ed.), InTech Europe, Croacia (2011)

Cortés, E., A. Dondero., H. Aros y C. Carlesi, Síntesis del líquido iónico bmin+HSO mediante una sola etapa, para aplicaciones en hidrometalurgia, Información Tecnológica: 21(3), 67-76 (2010).

Faúndez, C., y J. Valderrama, Avances sobre métodos de consistencia termodinámica de datos de equilibrio entre fases de mezclas binarias que contienen líquidos iónicos, Información Tecnológica: 24(4), 125-136 (2013). 
Ge, M. y otros cuatro autores, Densities and viscosities of 1-Butyl-3-methylimidazolium Trifluoromethanesulfonate $+\mathrm{H}_{2} \mathrm{O}$ binary mixtures at $\mathrm{T}=(303.15$ to 343.15) $\mathrm{K}$, J. Chem. Eng. Data: 53, 2408-2411 (2008).

Kaminsky, M., The concentration and temperature dependence of the viscosity of aqueous solutions of strong electrolytes. III. $\mathrm{KCl}, \mathrm{K}_{2} \mathrm{SO}_{4}, \mathrm{MgCl}_{2}, \mathrm{BeSO}_{4}$, and $\mathrm{MgSO}_{4}$ solutions, Z. Physik. Chem. Neue Floge: 12 , 206-231 (1957).

Khan, I., y otros cuatro autores, Probing the interactions between ionic liquids and water: Experimental and quantum chemical approach, J. Phys. Chem. B: 118, 1848-1860 (2014).

Martínez, M., y E. González, Mezclas binarias de líquido iónico con solvente molecular: predicción del volumen de exceso molar desde índices de refracción, Ciencia e ingeniería neogranadina: 21(2), 21-40 (2011).

Páez, M., A. Alvis y G. Arrázola, Efecto de la temperatura sobre la viscosidad de soluciones acuosas diluidas de Cloruro de 1-Butil-3-metil imidazolio [Bmim+][Cl], Información Tecnológica: 25(3), 185-190 (2014).

Páez, M., P.Cantero., y J. Peña, viscosidades de la mezcla n,n-Dimetilformamida +1-Butanol a diferentes temperaturas, Información Tecnológica: 23(3), 149-156 (2012).

Páez, M., M. Vergara., y E. Montes, Parámetros de interacción ión volumétricos del sistema 1-Butil-3-metil imidazolio trifluorometanosulfonato + agua a varias temperaturas, Información Tecnológica: 24(6), 47-58 (2013).

Passos, H., y otros nueve autores, Vapor-liquid equilibria of water + alkylimidazolium-based ionic liquids: measurements and perturbed-chain Statistical associating fluid theory modeling, Ind. Eng. Chem. Res: 53, 3737-3748 (2014).

Portacio, A., M. Páez., y J. Lafont, Efecto de la temperatura sobre las propiedades viscosimétricas y volumétricas de soluciones acuosas de pentanol, Revista Colombiana de Física: 43 (2), 622-626 (2011).

Seddon, R., A. Stark., y M. Torres, Viscosity and density of 1-Alkyl-3-methylimidazolium ionic liquids, pp 3449 ACS Symposium Series; American Chemical Society: Washington DC, USA ( 2002).

Seoane, R., y otros seis autores, Temperature dependence and structural influence on the thermophysical properties of eleven commercial ionic liquids, Ind. Eng. Chem. Res: 51, 2492-2504 (2012).

Shamsipur, M., y otros cuatro autores. Physical and electrochemical properties of ionic liquids 1-ethyl-3methylimidazolium tetrafluoroborate, 1-butyl-3-methylimidazolium trifluoromethanesulfonate and 1-butyl-1methylpyrrolidinium bis(trifluoromethylsulfonyl)imide, J. Mol. Liq : 157, 43-50 (2010).

Zhao, H., Viscosity B-coefficients and standard partial molar volumes of amino acids, and their roles in interpreting the protein (enzyme) stabilization, Biophysical Chemistry: 122, 157-183 (2006). 